\title{
Autophagy: A primer for the gastroenterologist/hepatologist
}

\author{
Christiane Sokollik MD, Michelle Ang MSc, Nicola L Jones MD FRCPC PhD
}

\begin{abstract}
C Sokollik, M Ang, NL Jones. Autophagy: A primer for the gastroenterologist/hepatologist. Can J Gastroenterol 2011; 25(12):667-674.
\end{abstract}

Autophagy is a conserved cellular pathway that maintains intracellular homeostasis by degrading proteins and cytosolic contents of eukaryotic cells. Autophagy clears misfolded and long-lived proteins, damaged organelles and invading microorganisms from cells, and provides nutrients and energy in response to exposure to cell stressors such as starvation. Defective autophagy has recently been linked to a diverse range of disease processes of relevance to gastroenterologists and hepatologists including Crohn's disease, pancreatitis, hepatitis and cancer. The present article provides an overview of the autophagy pathway and discusses gastrointestinal disease processes in which alterations in autophagy have been implicated. The clinical significance of autophagy as a potential therapeutic option is also discussed.

Key Words: Acute pancreatitis; Alpha-1-antitrypsin deficiency; Autophagy; Cancer; Crohn's disease; Cystic fibrosis; Ischemia-reperfusion injury

\section{L'autophagie : les notions élémentaires pour le gastroentérologue ou l'hépatologue}

L'autophagie est un mécanisme cellulaire conservé qui maintient l'homéostasie intracellulaire en dégradant les protéines et le contenu cytosolique des cellules eukaryotes. L'autophagie débarrasse la cellule des protéines mal pliées et trop vieilles, des organelles endommagées et des microorganismes envahissants. Elle fournit également à la cellule des nutriments et de l'énergie en réponse à l'exposition aux agresseurs cellulaires, tels que l'inanition. L'autophagie défectueuse a récemment été liée à divers processus pathologiques pertinents pour le gastroentérologue et l'hépatologue, y compris la maladie de Crohn, la pancréatite, l'hépatite et le cancer. Le présent article fournit un aperçu du mécanisme de l'autophagie et expose les processus des maladies gastrointestinales qui mettent en cause des modifications de l'autophagie. La signification clinique de l'autophagie comme option thérapeutique potentielle est également abordée.
$\mathrm{T}$ he terms 'autophagy' and 'autophagosome' were originally coined by De Duve and Wattiaux (1) in the 1960s to describe an intracellular digestive system closely related to lysosomes. Autophagy is a basal cellular pathway that maintains intracellular homeostasis by degrading proteins and cytosolic contents of eukaryotic cells. Autophagy clears misfolded and long-lived proteins, damaged organelles and invading microorganisms from cells, and provides nutrients and energy in response to exposure to cell stressors such as starvation. On initiation of autophagy, a cup-like isolation membrane that elongates to surround its cytoplasmic target is formed, and eventually seals to form a double-membrane vesicle known as an autophagosome. The autophagosome then fuses with lysosomes resulting in degradation of its contents (2) (Figure 1).

The three subtypes of autophagy - macroautophagy, microautophagy and chaperone-mediated autophagy - are distinguished by their delivery of material to the lysosome for degradation. Recently, the importance of the macroautophagy (hereafter referred to as autophagy) pathway in a variety of human diseases has been recognized. The present review focuses on the role of autophagy in gastrointestinal and liver diseases.

\section{THE AUTOPHAGY PATHWAY}

Autophagy-related (ATG) genes and proteins were first identified in yeast (3) and, because the autophagy machinery is an evolutionarily conserved process, their human counterparts were determined shortly thereafter (4). The formation of the autophagosome requires two ubiquitin-like conjugation systems (Figure 1). Complexes of ATG proteins, including ATG5, ATG12 and ATG16L1, are conjugated to the membrane of autophagosomes and are required for the 'maturation' of this compartment. The microtubule-associated protein 1 light chain 3 (LC3), the homologue of yeast ATG8, was identified as a mammalian autophagosome membrane protein, and is also a major tool to monitor autophagy in vitro and in vivo (5). LC3, which is located in the

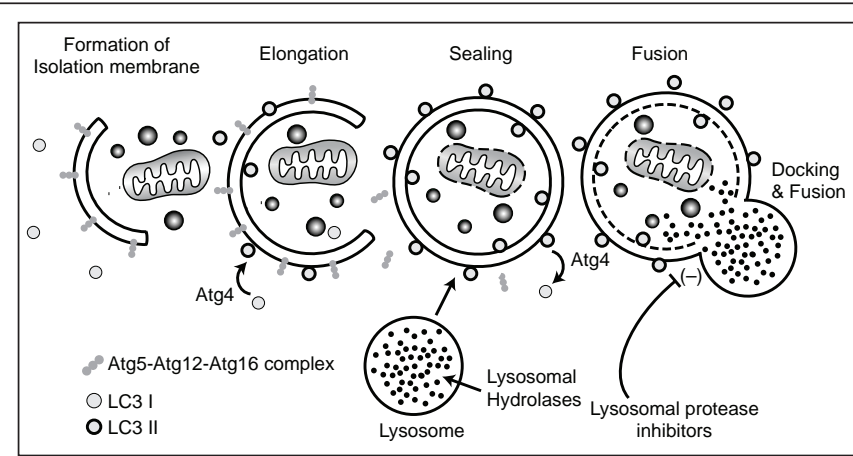

Figure 1) Autophagolysosome formation. On induction of autophagy, the isolation membrane or phagopore elongates and engulfs the cellular contents. Sealing of the tips leads to completion of the double-membrane autophagosome. The outer membrane then fuses with the lysosome resulting in degradation of its contents. Key elements of this process are the autophagy-related (ATG)12-ATG5-ATG16L complex, which recruits light chain (LC) 3 to the membrane, and ATG4, which controls the lipidation and recycling of LC3

cytoplasm, is recruited to the forming autophagosome membrane by interaction with the ATG12-ATG5-ATG16L complex. This complex is localized to the outer membrane of the autophagosome and supports the elongation of the isolation membrane and lipidation of LC3 (6-8). LC3 is linked to phosphatidylethanolamine (yielding a product known as LC3II), which is located on the inner and outer membranes of the autophagosome (9). LC3II remains associated with autophagosomes until its destruction at the autolysosomal stage. Thus, the amount of membrane-bound LC3II provides a good index of autophagy induction. LC3II located on the outer membrane can be deconjugated by ATG4, releasing LC3I for further use, whereas the LC3II on the inner membrane is degraded after fusion of the autophagosome with the lysosome.

Division of Gastroenterology, Hepatology and Nutrition, The Hospital for Sick Children; Departments of Paediatrics and Physiology, University of Toronto, Toronto, Ontario

Correspondence: Dr Nicola Jones, Division of Gastroenterology, Hepatology and Nutrition, The Hospital for Sick Children, University of Toronto,

555 University Avenue, Toronto, Ontario M5G 1X8. Telephone 416-813-7062, fax 416-813-6531, e-mail nicola.jones@sickkids.ca

Received for publication April 20, 2011. Accepted April 21, 2011 


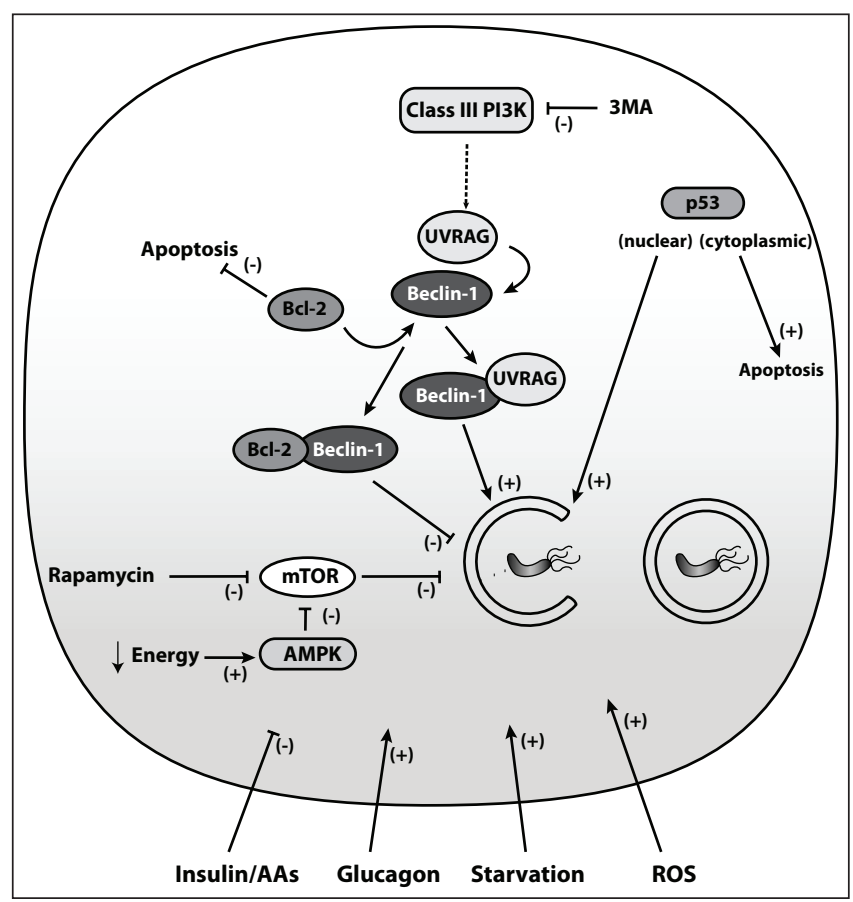

Figure 2) Autophagy regulation. Autophagy is embedded in a complex signaling network. Upstream inhibitors of autophagy include insulin and amino acids (AAs), whereas glucagon, starvation and reactive oxygen species (ROS) activate autophagy. Additional regulators of autophagy are the mammalian target of rapamycin (mTOR) and Beclin-1. The AMP-activated protein kinase (AMPK) senses energy deprivation and enhances autophagy via $m T O R$ inhibition. The main pharmacological mTOR inhibitor is rapamycin. Beclin-1 and ultaviolet radiation resistance-associated gene protein (UVRAG) activate autophagy, whereas binding of the anti-apoptotic Bcl-2/Bcl-Xl to Beclin-1 leads to inhibition of autophagy. The class III phosphatidylinositol-3-kinase (PI3K) regulates autophagy in concert with Beclin-1 and can be blocked by the pharmacological agent 3-methyladenine (3MA), thereby blocking autophagy. Both the tumour suppressor protein p53 and Bcl-2/Bcl-Xl connect autophagy with apoptosis

LC3II turnover is used to measure the extent of autophagy or autophagic flux. In cells treated with lysosomal protease inhibitors, degradation of inner membrane-bound LC3II is blocked and LC3II increases if the maturation of the autophagy machinery progresses normally (10). Knockout and knockdown of these ATGs are widely used methods to specifically inhibit autophagy to gain insight into the biological machinery surrounding the process.

The regulation of autophagy is complex and interconnects with multiple intracellular signalling networks at different levels. The catabolic hormone glucagon stimulates autophagy, while the anabolic hormone insulin and amino acids inhibit autophagy, indicating that autophagy is an energy-generating process $(11-13)$. The mammalian target of rapamycin (mTOR) is located downstream of the insulin receptor. mTOR negatively regulates autophagy so that activation of mTOR inhibits autophagy (Figure 2). On the other hand, rapamycin is a pharmacological inhibitor of mTOR and induces autophagy (14). Another mTOR-dependent pathway involves the energy-sensitive AMP-activated protein kinase, which is activated during energy deprivation and subsequently activates autophagy through mTOR inhibition (15). 3-methyladenine blocks autophagy via inhibition of the autophagy promoter class III phosphatidylinositol-3-kinase (PI3K) and is used in studies to block autophagy (16) (Figure 2). Furthermore, mTOR-dependent and -independent pathways involved in autophagy regulation are the inositol-monophosphatase, the c-Jun-N-terminalkinase and the death-associated protein kinase signalling cascades $(17-19)$.
In general, autophagy is regarded as a cell survival mechanism; however, autophagy-related cell death may also occur (20). A link between the autophagic and apoptotic machinery is found at the autophagosome initiation level where Beclin-1, an autophagy inducer, interacts with the anti-apoptotic protein $\mathrm{Bcl}-2$ or the $\mathrm{Bcl}-2$ homologue Bcl-Xl, preventing Beclin-1-dependent autophagy (21). For example, herpes viruses produce a $\mathrm{Bcl}-2$ homologue that promotes survival by preventing apoptotic cell death of virus-infected cells and suppressing autophagy (22). During starvation, the opposite takes place, ie, dissociation of Bcl-2 from Beclin-1 induces autophagy (23). Additional connections between autophagy and apoptosis include common stimuli such as reactive oxygen species and the tumour suppressor protein p53 (24) (Figure 2). However, the decisive factors distinguishing 'selfeating' autophagy from 'self-killing' apoptosis have yet to be entirely elucidated.

Autophagy was originally considered to be a nonselective process; however, studies performed in the past few years have demonstrated that autophagic adaptor proteins can target substrates for autophagic degradation. One such autophagy receptor is p62 (also known as SQSTM1/ sequestome-1), which delivers ubiquinated protein aggregates to the autophagosome (25) and also targets invading microorganisms for autophagy (26). There is evidence that p62-marked substrates are incorporated into the autophagosome via binding to LC3 (27). However, p62 can also localize to the endoplasmic reticulum (ER)associated autophagosome formation site independently of LC3 (28).

With the identification of key components and the tools to study the pathway, researchers have begun to investigate the significance of autophagy in various disorders. In the following sections, we highlight the recent knowledge regarding the role of autophagy in human gastrointestinal disorders including infections, inflammatory conditions and cancer.

\section{GASTROINTESTINAL AND HEPATIC INFECTIONS}

Some microorganisms invade cells in an attempt to generate a niche either in specialized compartments or free within the cytosol - to escape elimination by host defenses. However, within the cell, autophagy can target pathogens and restrict their growth. Autophagy of microbes is also known as xenophagy. Salmonella enteric serovar Typhimurium, which causes acute bloody diarrhea, and can also lead to severe invasive infections and sepsis in immunocompromised patients, replicates within Salmonella-containing vacuoles in host cells. Bacterial colonization of the cytosol is prevented by targeting Salmonella via the adapter protein p62 for autophagic degradation (26). In fact, increased cytosolic bacterial growth has been detected in autophagy-deficient cells (29). Autophagosomes can also target bacterial toxins to mitigate their toxic effects on host cells. During infection with Vibrio cholerae, which causes life-threatening diarrhea, the autophagic pathway protects cells against the pore-forming exotoxin $V$ cholerae cytolysin (VCC). VCC-intoxicated cells induce autophagy to degrade VCC and promote survival. In contrast, cells with defective autophagy are more sensitive to the cytotoxicity of VCC (30).

The gastric pathogen Helicobacter pylori, which causes peptic ulcer disease and gastric cancer, expresses various virulence factors to mediate disease. One of these factors, the vacuolating cytotoxin VacA, is produced by some strains, and its presence is associated with more severe disease. VacA is internalized within the host cell, inducing the formation of vacuoles and altering host cell signalling (31). In addition, $H$ pylori can survive intracellularly in VacA-dependent vacuoles in vitro (32). Short-term exposure to VacA also induces the formation of autophagosomes in gastric epithelial cells in vitro (33). A portion of the toxin is found within these autophagosomes indicating that autophagy targets the toxin. When autophagy is disrupted in host cells, degradation of the toxin is prevented and toxin-mediated cellular vacuolization is increased, indicating that autophagy can eliminate the toxin and mitigate its detrimental effects in host cells.

These studies indicate that autophagy acts as an innate host defense against infectious agents. However, some microorganisms have 
developed mechanisms to manipulate the pathway, thereby avoiding clearance. For example, microbes may prevent the initiation of autophagy, block autophagosome maturation or even usurp the autophagosome to create an intracellular niche (34). Shigella, which causes dysentery and whose toxin-producing strains are associated with the development of hemolytic uremic syndrome, can prevent detection by the autophagic machinery. Shigella moves within the cell and promotes cell-to-cell spreading by actin-based motility. The surface component VirG, which is necessary for Shigella's actin-dependent motility, can be recognized and targeted for autophagy by the ATG5 protein. However, Shigella also secretes the bacterial protein IcsB, which camouflages $\mathrm{VirG}$, thereby enabling Shigella to escape ATG5 detection and subsequent autophagic clearance (35).

Viruses use host cells and their resources for their own life cycle. The hepatitis B virus replicates by inducing autophagy and the development of autophagic vacuoles, whereas it simultaneously prevents maturation to autolysosomes, thus avoiding lysosomal degradation (36). Similarly, hepatitis $\mathrm{C}$ virus (HCV) replication is dependent on autophagy, illustrated by knockdown of Beclin-1, which reduces $\mathrm{HCV}$ production, enhances cell death and prevents persistent infection (37). Interestingly, autophagic proteins are needed to initiate HCV RNA translation, but not to maintain it, suggesting a contribution of autophagic proteins in the delivery of HCV RNA to the translational apparatus (38).

These examples demonstrate the potential complexity of hostpathogen interplay in the autophagy pathway during gastrointestinal or hepatic infections. Therefore, careful consideration of this complexity is important for the development of novel therapies that, depending on the specific context, could either promote or inhibit the pathway to ensure beneficial effects for the host.

\section{CROHN'S DISEASE}

In 2007, a genome-wide association study (39) identified a nonsynonymous single-nucleotide polymorphism (rs2241880) causing a threonine to alanine substitution at position 300 (T300A) of the ATG16L1 gene as a susceptibility variant for Crohn's disease (CD). The variant is associated with the WD repeat region of ATG16L1, the function of which is unknown. As described above, ATG16L forms a complex with ATG5 and ATG12, which is necessary for autophagosome formation. Several groups have attempted to delineate the potential functional relevance of this allele in mediating $\mathrm{CD}$. In one study, decreased efficiency of antibacterial autophagy was observed in human cell cultures overexpressing the ATG16L1 CD variant (40). Another study determined that fetal hepatic macrophages obtained from ATG16L knockout mice produced higher levels of the inflammatory cytokines interleukin-1-beta and interleukin-18 in response to bacterial lipopolysaccharide exposure compared with control macrophages. In addition, mice transplanted with the ATG16L knockout macrophages were more susceptible to dextran sulphate sodium (DSS)-induced acute colitis (41). Cadwell et al (42) generated mice with reduced ATG16L protein (hypomorphs) to address the role of ATG16L1. These mice developed changes in the Paneth cell granule exocytosis pathway, which lacked lysozymes in the mucus layer of the ileum (Figure 3). Paneth cells are located in the crypts of Lieberkühn of the small intestine, and secrete lysozymes and antimicrobial peptides into the lumen of the crypt to protect against pathogenic invasion. These Paneth cell abnormalities were also identified in human tissues from CD patients with the risk allele of ATG16L1 (42). Although these studies did not specifically investigate the endogenously expressed $\mathrm{CD}$ variant, the findings suggest that the $\mathrm{CD}$ variant may alter autophagy and host inflammatory pathways in response to microbes or their products.

The etiology of $\mathrm{CD}$ is multimodal and involves genetic, immunological and environmental factors. The multihit hypothesis - that genetically susceptible individuals primed by an unknown event develop CD after another incident occurred - may explain the fact that only a small percentage of individuals with the common disease

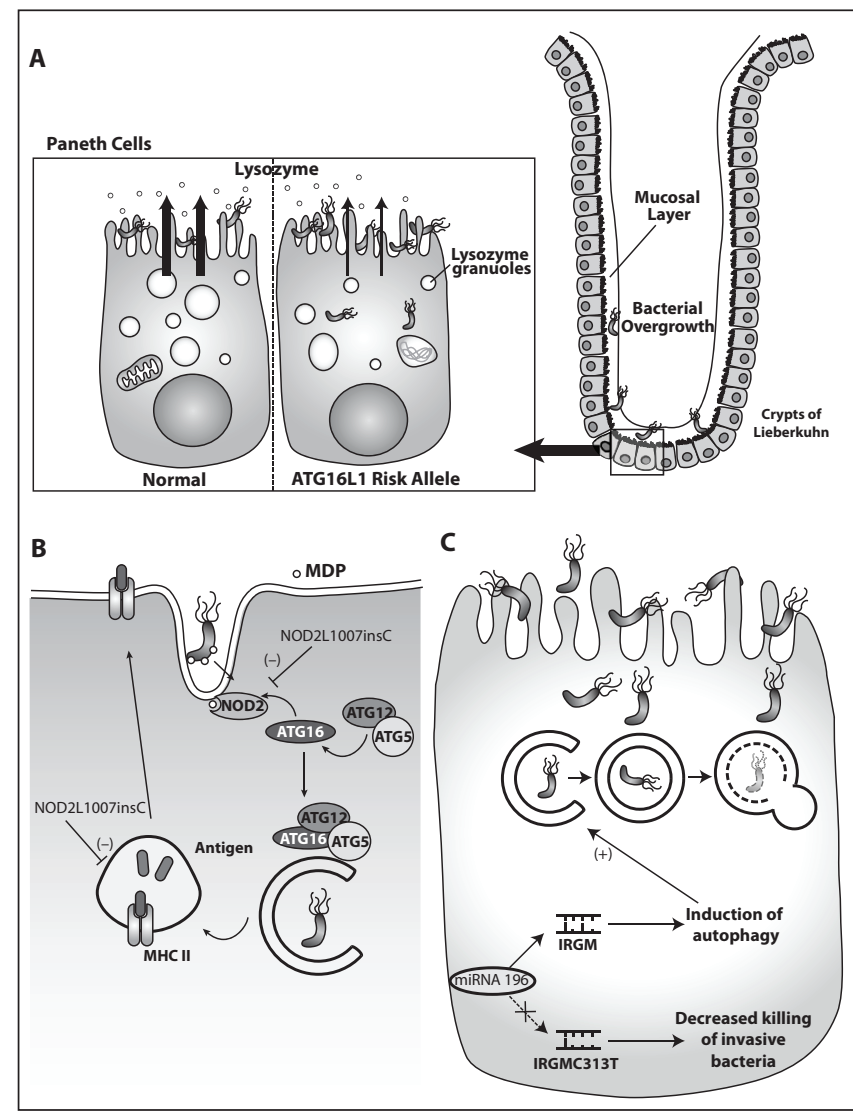

Figure 3) Autophagy in Crohn's disease. A Paneth cells with the autophagyrelated (ATG) $16 \mathrm{~L}$ risk allele show structural and functional abnormalities. They exhibit disorganized and diminished lysozyme granules and degraded mitochondria resulting in reduced lysozymes in the mucosal layer potentially causing bacterial overgrowth. B Nucleotide-binding oligomerization domain containing 2 (NOD2) senses bacterial muramyl dipeptide (MDP) and recruits ATG16L to the cellular membrane to initiate autophagy of intracellular bacteria. Mutant NOD2L1007insC has reduced autophagy in response to MDP. Additionally, the NOD2-supported antigen presentation via major histocompatibility complex class II (MHC II) in dendritic cells with the risk alleles is defective and results in impaired activation of CD4 T cells. C Immunity-related GTPase family, M (IRGM) activates autophagy for xenophagy. The Crohn's disease risk allele IRGM C313T messenger RNA lacks the binding site for microRNA (miRNA)-196, leading to upregulation of IRGM, which results in decreased efficacy of autophagy and increased intracellular bacterial survival

variant ATG16L are actually affected by CD. In a follow-up to their previous study, Cadwell et al (43) found that mice with reduced levels of ATG16L1 raised in a germ-free facility lacked the Paneth cell abnormalities until they were infected with a specific murine norovirus. Subsequent treatment with DSS induced severe colitis with some of the typical features of the transmural colitis seen in CD patients, whereas uninfected ATG16L mutant mice and wild-type mice showed only focal ulcerating DSS colitis. In addition, broadspectrum antibiotic treatment prevented DSS-induced colitis, indicating a requirement for a bacterial component after the viral trigger (43). Another feature highlighted by this study is the possible contribution of viral infections to the pathogenesis of CD.

ATG16L T300A is not a disease-causing polymorphism; therefore, the functional relevance of this variant also needs to be evaluated in the context of other risk alleles. Nucleotide-binding oligomerizationdomain-containing 2 (NOD2), an intracellular pattern recognition receptor for bacterial muramyl-dipeptide (MDP), was the first gene associated with CD (44). The most prevalent NOD2 polymorphism 


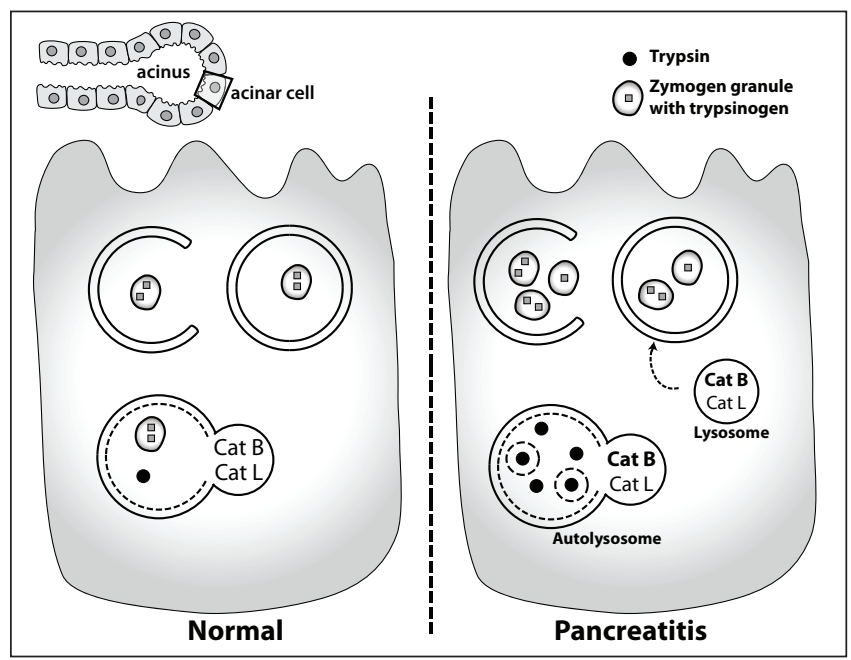

Figure 4) Autophagy in pancreatitis. Autophagy is induced during pancreatitis, but the degradation of autolysosomes is impaired with accumulation of large vacuoles. Reduced activity of the lysosomal cathepsins (Cat) $L$ and $B$ was detected in autophagosomes, with $C$ at $L$ more greatly affected than Cat B. The imbalance between the lysosomal hydrolases Cat B and Cat $L$ increases the intra-acinar trypsin leading to cell necrosis and inflammation

L1007insC in CD is a frameshift mutation, which results in a truncated NOD2 protein. A logical step after sensing intracellular bacteria via NOD-like receptors would be to induce xenophagy. Indeed, Travassos et al (45) demonstrated that both bacteria and NOD ligands trigger autophagy. In addition, NOD2 interacted with and recruited ATG16L to the plasma membrane at the bacterial entry site to initiate autophagy. In cells expressing the most common CD-associated NOD2 mutation, mutant NOD2 was retained in the cytosol and failed to recruit ATG16L. As a consequence, autophagosome formation was impaired (45) (Figure 3). Homer et al (46) confirmed that stimulation with MDP increases autophagy. In addition, they demonstrated that NOD2-mediated autophagy has antibacterial effects against $S$ typhimurium, which was defective in cells with CD-associated NOD2 mutations. This NOD2-dependent antibacterial effect was also reduced in epithelial cells with ATG16L1 knockdown and reconstitution of CD, but not in the wild-type variant of ATG16L1. In contrast, peripheral blood moncytes or dendritic cells from individuals heterozygous or homozygous for the CD variant showed no deficit in NOD2-dependent bacterial killing (46). These findings support the functional connection between the CD risk alleles NOD2L1007insC and ATG16L T300A in a cell-specific, intracellular response to bacteria with failed bacterial clearance by autophagy.

In addition to its involvement in the innate immune response, autophagy also plays a role in the adaptive immune response. Autophagosomes fuse with multivesicular major histocompatibility complex (MHC) class II-loading compartments to present their contents on the outer cell membrane for recognition by antigen-specific $\mathrm{CD}^{+} \mathrm{T}$ cells (47). Cooney et al (48) found that in dendritic cells exposed to MDP, NOD2 induced autophagy and promoted MHC class II antigen presentation. However, this process was defective in dendritic cells from CD patients with either ATG16L or NOD2 risk alleles (48) (Figure 3).

An additional risk allele for CD affects another autophagy gene, IRGM (immunity-related GTPase family, M). IRGM controls intracellular Mycobacterium tuberculosis survival via the induction of autophagy (49), and IRGM expression levels regulate xenophagy of $S$ typhimurium (50). The IRGM C313T risk allele for CD results in a messenger RNA (mRNA) that loses its binding site for microRNA (miRNA)-196 (51). miRNAs are capable of post-translational gene silencing by binding to complementary mRNA, thereby regulating gene expression. In biopsies obtained from inflamed intestinal tissue of CD patients, miRNA-196 was found to be overexpressed and could downregulate IRGM only in CD patients without the risk allele (51). Paradoxically, the enhanced expression of IRGM C313T by lack of miRNA-196 regulation decreased the host cell response to infection with adherent-invasive Escherichia coli (Figure 3).

All three susceptibility variants for CD - ATG16L T300A, NOD2L1007insC and IRGM C313T - in some way link autophagy with microorganisms and distorted host cell responses. Despite the potential involvement of a multitude of environmental and genetic risk factors, the pathogenesis of CD may ultimately be the result of a few pathways that are simply affected at different stages and in different combinations.

\section{ACUTE PANCREATITIS}

Pancreatic proenzymes are stored in zymogen granules of acinar cells and enzyme inhibitors, such as the serine protease inhibitor Kazal-type 1, bind prematurely activated trypsin to prevent autodigestion of the pancreas. In acute pancreatitis, these protective mechanisms are exhausted, and activated trypsin within acinar cells leads to cell necrosis with further release of additional activated enzymes. Recently, several groups investigated the potential role of autophagy in the pathogenesis of acute pancreatitis. Hashimoto et al (52) showed that induction of acute pancreatitis with supraphysiological doses of cerulein, a cholecystokinin analogue, induces autophagy in acinar cells and that acinar cell-specific ATG5 knockout mice with disrupted autophagy had less severe pancreatitis and less conversion of trypsinogen to trypsin in their acinar cells (52). The authors suggested that autophagosomes engulf zymogen granules and deliver them to low $\mathrm{pH}$ and cathepsin B-containing lysosomes where the activation of trypsinogen occurs. However, increased autophagy also occurs in acinar cells upon starvation without a corresponding increase in activation of trypsinogen (53). In another study, Mareninova et al (53) demonstrated that defective autophagy was induced during acute pancreatitis. In this study, impaired activity of the lysosomal cathepsins L and B was detected in autophagosomes, with cathepsin L more greatly affected than cathepsin B. The authors hypothesized that because cathepsin B converts trypsinogen to trypsin, and cathepsin L degrades trypsin, this imbalance would promote increased active trypsin within cells (Figure 4). However, the underlying mechanisms responsible for these observations remain unknown.

In contrast to these studies, Grasso et al (54) provided evidence that selective zymogen autophagy is a protective mechanism in acute pancreatitis because knockdown of vacuole membrane protein-1 (VMP1), an autophagy-related protein that induces autophagy, prevented pancreatic acinar cell death in experimental models (54). In addition, VMP1 was undetectable in normal human acini but present in tissue affected by pancreatitis in association with autophagosome formation.

These apparently contradictory studies suggest a major role for autophagy in acute pancreatitis. However, additional studies are needed to determine whether there is variation in specific types of autophagy during pancreatitis, some of which may be protective and others harmful, thereby providing a potential explanation for these divergent results as well as directing therapeutic options.

\section{CANCER}

Autophagy can have diametrically opposing effects in tumorigenesis, playing a role in both tumour suppression and promotion (55). Autophagosomes can engulf and eliminate damaged organelles that produce reactive oxygen species and cause DNA damage. Similarly, autophagosomes can target protein aggregates that may modulate protumorigenic signal transduction pathways. Both of these mechanisms can prevent tumour development. In contrast, a shortage of nutrients within the tumour environment can trigger autophagy to provide nutrients and energy, thereby promoting survival of tumour cells and supporting their progression. 
Initial evidence supporting a role for autophagy in tumour suppression came from studies in Beclin-1 deficient mice. Aging Beclin-1 $1^{+-}$ mutant mice with impaired autophagy developed spontaneous tumours including hepatocellular carcinoma (HCC) (56). Consistent with these findings, human HCC tissue samples showed lower Beclin-1 expression than nontumour tissue (57), indicating that autophagy has tumour suppressor functions and that Beclin-1 is a haploinsufficient tumour suppressor. Similarly, monoallelic mutations in the ultraviolet radiation-resistance-associated gene (UVRAG), an inducer of autophagy through binding to Beclin-1, are found in human colon cancer (58) and in gastric carcinomas with microsatellite instability (59). Liang et al (60) demonstrated that overexpression of UVRAG induces autophagy and suppresses the proliferation and tumorigenicity of human colon cancer cells. Additionally, mice inoculated with UVRAG mutant tumour cells developed larger tumour masses than mice inoculated with tumour cells in which UVRAG was replaced (60).

In both human gastric adenocarcinomas and HCC tissue samples, $\mathrm{mTOR}$ expression is increased, and tumour growth and angiogenesis is constricted in experimental models following treatment with rapamycin, which inhibits mTOR and induces autophagy $(61,62)$. These findings also suggest that rapamycin, which blocks mTOR activation, may have chemotherapeutic potential in gastrointestinal and liver tumours. In support of this contention, a recent study reported improved survival after liver transplantation for HCC in patients treated with a rapamycin-based immunosuppression protocol (63).

In contrast to its tumour-suppressing effects, autophagy may also help tumour cells survive when oxygen and nutrients are diminished. Tumorigenic cells with defective apoptosis but functioning autophagy display a survival advantage under ischemic conditions compared with cells with blocked autophagy (64). Thus, at first, autophagy may protect against tumour initiation, whereas later in established tumours, it promotes survival.

Due to the opposing effects of autophagy in tumorigenesis, targeting this pathway in anticancer therapy may be particularly difficult. For example, inhibition of autophagy increased sensitivity to radiation in radioresistant human cancer cell lines in one study (65), whereas another study showed that induction of autophagy radiosensitized prostate cancer cells (66). The tumour suppressor p53 induces autophagy and apoptosis (67). Treatment of lymphoma cells with the p53 stimulator tamoxifen led to tumour apoptosis and increased autophagy in surviving cells. Cotreatment with chloroquine, a pharmacological inhibitor of autophagy, enhanced p53-dependent apoptosis and tumour regression by blocking autophagy (68). In this instance, it can be reasoned that a combination therapy of apoptosis-activating and autophagy-blocking agents is a very potent treatment strategy. Therefore, autophagy modulation as an adjuvant to standard chemotherapy may improve efficacy by shifting the balance from autophagy to apoptosis, but needs to be tumour and tumour-stage specific (69).

\section{PROTEIN FOLDING DISORDERS}

\section{Alpha-1-antitrypsin deficiency}

The acute-phase reactant alpha-1-antitrypsin (A1AT) is a protease inhibitor produced in hepatocytes, which protects tissue from neutrophil elastase in particular. A1AT deficiency is primarily associated with respiratory complications, but it is also the most common genetic cause of liver disease in children. The classical $\mathrm{Z}$ mutation leads to a misfolded protein, which accumulates in the ER and activates nuclear factor kappa B, ER caspases and BAP31. These responses may explain the hepatic inflammation and inflammation-associated carcinogenesis in patients with A1AT deficiency (70). However, not all affected homozygotes develop liver disease and additional disease modifiers may play a role. There is convincing evidence that autophagy plays a role in the pathophysiology of A1AT deficiency because liver tissue from patients with A1AT deficiency showed increased autophagosome formation, and degradation of A1AT mutants was reduced when autophagy was blocked (71). Treatment of $\mathrm{Z}$ mutant mice with carbamazepine, an established antiepileptic drug with autophagy-inducing

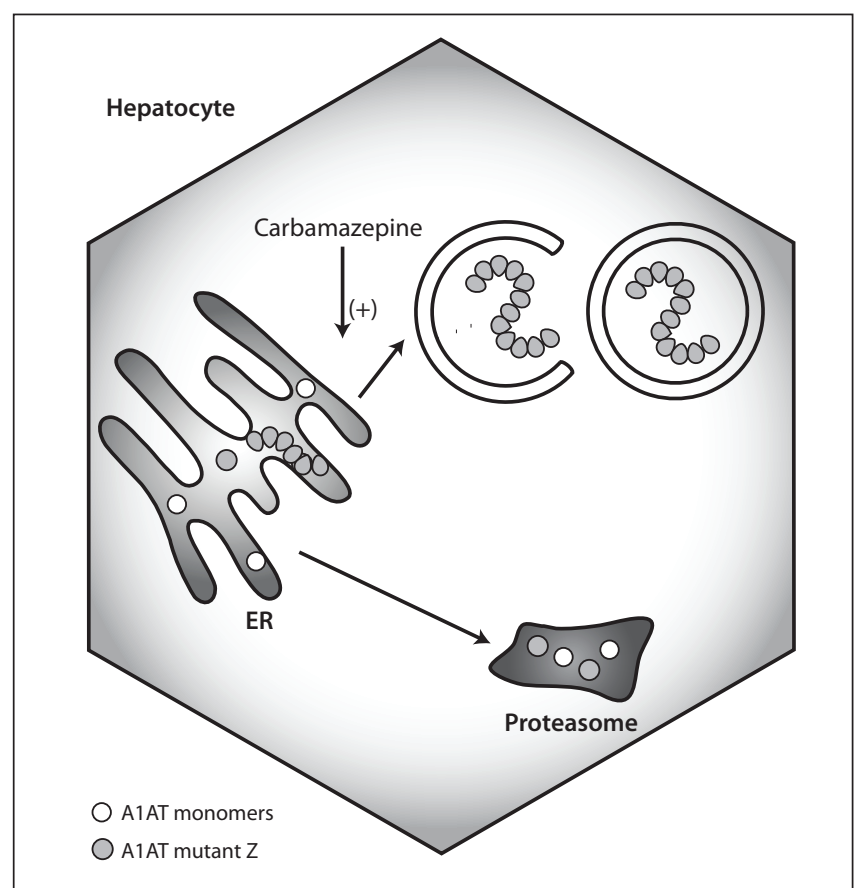

Figure 5) Autophagy in alpha-1-antitrypsin (A1AT) deficiency. The misfolded mutant A1AT forms polymers that accumulate in the endoplasmatic reticulum (ER), which causes ER stress and inflammation. Monomers can be degraded in proteasomes whereas polymer degradation takes place in autophagolysosomes. Enhancing autophagy with carbamazepine increases the disposal of mutant A1AT and subsequently ameliorates its hepatotoxicity

properties, promoted the degradation of mutant A1AT and even decreased hepatic fibrosis (72) (Figure 5). This intriguing study suggests that enhancing autophagy may be a promising pharmacological intervention in A1AT. However, rapamycin failed to show enhanced disposal of mutant A1AT, which may suggest a role for an mTORindependent pathway. Proteasomal clearance may also be part of the carbamazepine effect because proteasomal inhibitors lessened the degree of carbamazepine-induced degradation of A1AT. More studies are needed to clarify the role of autophagy in A1AT deficiency because carbamazepine seems to influence other intracellular mechanisms. In addition, despite the favourable safety profile of carbamazepine, its feasibility needs to be evaluated if the high doses required in mice are also required in humans.

\section{CYSTIC FIBROSIS}

Cystic fibrosis (CF) is caused most commonly by a $\triangle F 508$ mutation in the CF transmembrane conductance regulator (CFTR) gene, which results in a misfolded protein that does not reach the cell membrane and is degraded rapidly. Decreased LC3II and Beclin-1 protein levels were observed in nasal polyps from severely affected CF patients, in lung tissue from CF mice and in human CF bronchial epithelial cells. These levels could not be increased by autophagy induction via starvation or rapamycin, suggesting defective autophagy (73). Previously, it was shown that tissue transglutaminase is upregulated in CF (74), and that there is a link between oxidative stress and inflammation in $\mathrm{CF}$ (75). Derived from these findings, treatment with cystamine - a transglutaminase inhibitor, and N-acetyl-L cysteine - an antioxidant, increased Beclin-1 levels, rescued autophagy and ameliorated lung injury by preventing the damaging aggresome formation of the CFTR $\Delta \mathrm{F} 508$ protein (73). However, it is not known whether deficient autophagy is involved in mediating the disease found in other organs such as the pancreas or the liver; this is an area of interest for future investigation. 


\section{ISCHEMIA-REPERFUSION INJURY}

Ischemia-reperfusion injury (IRI) occurs during liver transplantation or hepatic resection when blood supply is disrupted and subsequently restored. Damaged mitochondria with impaired oxidative phosphorylation lose their membrane potential, produce reactive oxygen species and release proapoptotic factors. The innate immune system is especially activated during reperfusion and, thus, the inflammatory response leads to aggravated organ injury. In an in vivo model of ischemia-reperfusion with temporary clamping of liver vessels, pretreatment with the autophagy-inducing agent cisplatin led to a reduced expression of the inflammatory mediators tumour necrosis factor-alpha and interleukin-6, and protected mice from liver injury as assessed by transaminase levels (76). In the livers of the cisplatin-treated mice, Beclin-1 and LC3 levels were increased, and ultrastructural analysis with electron microscopy showed autophagy of mitochondria (also known as mitophagy). In contrast, in a study of prolonged cold ischemia of rat livers using University of Wisconsin solution, the inhibition of autophagy with wortmannin increased the survival rate of the rat liver recipients (77). Comparing these two studies highlights the difficulty of translating the results of cell culture or animal models into clinical practice. Many factors may explain the apparently conflicting results including the differences in modelling ischemia-reperfusion with variable durations of ischemia, warm versus cold ischemia, preservation solution with or without amino acids, and potential autophagyindependent effects of the pharmacological agents.

More insight into the role of autophagy in IRI may be obtained as our understanding of ischemic preconditioning (IP) evolves. The beneficial effects of IP were first described in the heart. IP with interruption of the blood supply followed by reperfusion protects the myocardium from a subsequent ischemic insult (78). In a study of patients who underwent hepatic resection of colon cancer metastasis (79), increased LC3 expression and autophagosome formation was detected in liver biopsies following IP compared with biopsies taken before IP. Conflicting results were found in studies that assessed the impact of IP on postoperative liver function, morbidity and mortality rates (80-82). Thus, additional studies are needed to determine whether the increase in autophagy after IP has a protective role in IRI (83).

\section{THERAPEUTIC CONSIDERATIONS}

As outlined above, given the importance of autophagy in a variety of diseases, a great deal of interest has centred on the development of potential therapies to modulate this pathway. Thus, the gastroenterologist or hepatologist should be aware of these developments to stay abreast of advances in treatment modalities.

In large screenings, it became evident that the mechanism of action of some previously approved drugs involved autophagy (84). The antiarrhythmic drug amiodarone (a $\mathrm{Ca}^{2+}$ channel blocker), and loperamide (an opioid-receptor agonist) may induce autophagy through regulation of intracellular $\mathrm{Ca}^{2+}$. The glucocorticoid dexamethasone induces autophagy in hematological cancer cells and may support autophagy-dependent cell death in malignancies (85). Above, we discussed a possible therapeutic broadening of the mood stabilizer carbamazepine, which induces autophagy independent of mTOR, similar to lithium and valproic acid (17). Rapamycin, an mTOR inhibitor, is used as an immunosuppressive drug in solid organ transplantation, especially if the transplantation is associated with malignancies such as HCC or post-transplant lymphoproliferative disease (86), but its strong immunosuppressive potential with the risk of side effects limits its use in other settings. A combination of mTORdependent and -independent autophagy enhancers may maximize the benefits of autophagy induction and simultaneously allow reduction of drug doses, thereby decreasing side effects (87). In anticancer treatment, the combination of antineoplastic therapies with modulators of autophagy may be beneficial but, as discussed above, the effects of autophagy during tumorigenesis are complex and depend on, among others, the tumour type and stage. As we gain a deeper understanding of the mechanisms regulating cargo-specific autophagic processes and disease, the potential to develop molecules that specifically target these components while minimizing the effect on other pathways offers great therapeutic potential.

\section{CONCLUSION}

Alterations in the autophagy pathway are increasingly implicated in a variety of gastroenterological, hepatological and pancreatic disorders. There is a need for increased understanding of the mechanisms underlying these observations, suitable models to study the pathway in disease and the potential benefit of pharmacological manipulation of the pathway before clinical trials can be safely performed. Although initial reports about the benefit of autophagymodulating drugs are promising, there is a need to develop highly selective drugs that target specific components of the autophagy pathway and are suitable for clinical use.

ACKNOWLEDGEMENTS: NLJ is supported by CIHR (No. 178886) and by the Crohn's and Colitis Foundation of Canada.

\section{REFERENCES}

1. de Duve C, Wattiaux R. Functions of lysosomes. Annu Rev Physiol 1966;28:435-92.

2. Seglen PO, Bohley P. Autophagy and other vacuolar protein degradation mechanisms. Experientia 1992;48:158-72.

3. Matsuura A, Tsukada M, Wada Y, Ohsumi Y. Apg1p, a novel protein kinase required for the autophagic process in Saccharomyces cerevisiae. Gene 1997;192:245-50.

4. Mizushima N, Sugita H, Yoshimori T, Ohsumi Y. A new protein conjugation system in human. The counterpart of the yeast Apg12p conjugation system essential for autophagy. J Biol Chem 1998;273:33889-92.

5. Kabeya Y, Mizushima N, Ueno T, et al. LC3, a mammalian homologue of yeast Apg8p, is localized in autophagosome membranes after processing. EMBO J 2000;19:5720-8.

6. Mizushima N, Yamamoto A, Hatano M, et al. Dissection of autophagosome formation using Apg5-deficient mouse embryonic stem cells. J Cell Biol 2001;152:657-68.

7. Mizushima N, Kuma A, Kobayashi Y, et al. Mouse Apg16L, a novel WD-repeat protein, targets to the autophagic isolation membrane with the Apg12-Apg5 conjugate. J Cell Sci 2003;116:1679-88.

8. Fujita N, Itoh T, Omori H, Fukuda M, Noda T, Yoshimori T. The Atg16L complex specifies the site of LC3 lipidation for membrane biogenesis in autophagy. Mol Biol Cell 2008;19:2092-100.

9. Ichimura Y, Kirisako T, Takao T, et al. A ubiquitin-like system mediates protein lipidation. Nature 2000;408:488-92.

10. Klionsky DJ, Abeliovich H, Agostinis P, et al. Guidelines for the use and interpretation of assays for monitoring autophagy in higher eukaryotes. Autophagy 2008;4:151-75.

11. Deter RL, Baudhuin P, de Duve C. Participation of lysosomes in cellular autophagy induced in rat liver by glucagon. J Cell Biol 1967;35:C11-C16.

12. Pfeifer U. Inhibition by insulin of the formation of autophagic vacuoles in rat liver. A morphometric approach to the kinetics of intracellular degradation by autophagy. J Cell Biol 1978;78:152-67.

13. Mortimore GE, Schworer CM. Induction of autophagy by amino-acid deprivation in perfused rat liver. Nature 1977;270:174-6.

14. Noda T, Ohsumi Y. Tor, a phosphatidylinositol kinase homologue, controls autophagy in yeast. J Biol Chem 1998;273:3963-6.

15. Inoki K, Zhu T, Guan KL. TSC2 mediates cellular energy response to control cell growth and survival. Cell 2003;115:577-90.

16. Seglen PO, Gordon PB. 3-methyladenine: Specific inhibitor of autophagic/lysosomal protein degradation in isolated rat hepatocytes. Proc Natl Acad Sci USA 1982;79:1889-92

17. Sarkar S, Floto RA, Berger Z, et al. Lithium induces autophagy by inhibiting inositol monophosphatase. J Cell Biol 2005;170:1101-11.

18. Wei Y, Pattingre S, Sinha S, Bassik M, Levine B. JNK1-mediated phosphorylation of $\mathrm{Bcl}-2$ regulates starvation-induced autophagy. Mol Cell 2008;30:678-88.

19. Zalckvar E, Berissi H, Mizrachy L, et al. DAP-kinase-mediated phosphorylation on the $\mathrm{BH} 3$ domain of beclin 1 promotes dissociation of beclin 1 from Bcl-XL and induction of autophagy. EMBO Rep 2009;10:285-92. 
20. Levine B, Yuan J. Autophagy in cell death: An innocent convict? J Clin Invest 2005;115:2679-88.

21. Pattingre S, Tassa A, Qu X, et al. Bcl-2 antiapoptotic proteins inhibit Beclin 1-dependent autophagy. Cell 2005;122:927-39.

22. Liang C, E X, Jung JU. Downregulation of autophagy by herpesvirus Bcl-2 homologs. Autophagy 2008;4:268-72.

23. Maiuri MC, Le Toumelin G, Criollo A, et al. Functional and physical interaction between $\mathrm{Bcl}-\mathrm{X}(\mathrm{L})$ and a $\mathrm{BH} 3$-like domain in Beclin-1. EMBO J 2007;26:2527-39.

24. Maiuri MC, Tasdemir E, Criollo A, et al. Control of autophagy by oncogenes and tumor suppressor genes. Cell Death Differ 2009;16:87-93.

25. Bjorkoy G, Lamark T, Brech A, et al. p62/SQSTM1 forms protein aggregates degraded by autophagy and has a protective effect on huntingtin-induced cell death. J Cell Biol 2005;171:603-14.

26. Zheng YT, Shahnazari S, Brech A, Lamark T, Johansen T, Brumell JH. The adaptor protein p62/SQSTM1 targets invading bacteria to the autophagy pathway. J Immunol 2009;183:5909-16.

27. Ichimura Y, Kumanomidou T, Sou YS, et al. Structural basis for sorting mechanism of p62 in selective autophagy. J Biol Chem 2008;283:22847-57.

28. Itakura E, Mizushima N. p62 targeting to the autophagosome formation site requires self-oligomerization but not LC3 binding. J Cell Biol 2011;192:17-27.

29. Birmingham CL, Smith AC, Bakowski MA, Yoshimori T, Brumell JH. Autophagy controls Salmonella infection in response to damage to the Salmonella-containing vacuole. J Biol Chem 2006;281:11374-83.

30. Saka HA, Gutierrez MG, Bocco JL, Colombo MI. The autophagic pathway: A cell survival strategy against the bacterial pore-forming toxin Vibrio cholerae cytolysin. Autophagy 2007;3:363-5.

31. Ceponis PJ, Jones NL. Modulation of host cell signal transduction pathways by Helicobacter pylori infection. Can J Gastroenterol 2005;19:415-20.

32. Terebiznik MR, Vazquez CL, Torbicki K, et al. Helicobacter pylori VacA toxin promotes bacterial intracellular survival in gastric epithelial cells. Infect Immun 2006;74:6599-614.

33. Terebiznik MR, Raju D, Vazquez CL, et al. Effect of Helicobacter pylori's vacuolating cytotoxin on the autophagy pathway in gastric epithelial cells. Autophagy 2009;5:370-9.

34. Deretic V, Levine B. Autophagy, immunity, and microbial adaptations. Cell Host Microbe 2009;5:527-49.

35. Ogawa M, Yoshimori T, Suzuki T, Sagara H, Mizushima N, Sasakawa C. Escape of intracellular Shigella from autophagy. Science 2005;307:727-31.

36. Sir D, Tian Y, Chen WL, Ann DK, Yen TS, Ou JH. The early autophagic pathway is activated by hepatitis $B$ virus and required for viral DNA replication. Proc Natl Acad Sci USA 2010;107:4383-8.

37. Shrivastava S, Raychoudhuri A, Steele R, Ray R, Ray RB. Knockdown of autophagy enhances the innate immune response in hepatitis C virus-infected hepatocytes. Hepatology 2011;53:406-14.

38. Dreux M, Gastaminza P, Wieland SF, Chisari FV. The autophagy machinery is required to initiate hepatitis $\mathrm{C}$ virus replication. Proc Natl Acad Sci USA 2009;106:14046-51.

39. Hampe J, Franke A, Rosenstiel P, et al. A genome-wide association scan of nonsynonymous SNPs identifies a susceptibility variant for Crohn disease in ATG16L1. Nat Genet 2007;39:207-11.

40. Kuballa P, Huett A, Rioux JD, Daly MJ, Xavier RJ. Impaired autophagy of an intracellular pathogen induced by a Crohn's disease associated ATG16L1 variant. PLoS One 2008;3:e3391.

41. Saitoh T, Fujita N, Jang MH, et al. Loss of the autophagy protein Atg16L1 enhances endotoxin-induced IL-1beta production. Nature 2008;456:264-8.

42. Cadwell K, Liu JY, Brown SL, et al. A key role for autophagy and the autophagy gene Atg1611 in mouse and human intestinal Paneth cells. Nature 2008;456:259-63.

43. Cadwell K, Patel KK, Maloney NS, et al. Virus-plus-susceptibility gene interaction determines Crohn's disease gene Atg16L1 phenotypes in intestine. Cell 2010;141:1135-45.

44. Hugot JP, Chamaillard M, Zouali H, et al. Association of NOD2 leucine-rich repeat variants with susceptibility to Crohn's disease. Nature 2001;411:599-603.

45. Travassos LH, Carneiro LA, Ramjeet M, et al. Nod1 and Nod2 direct autophagy by recruiting ATG16L1 to the plasma membrane at the site of bacterial entry. Nat Immunol 2010;11:55-62.
46. Homer CR, Richmond AL, Rebert NA, Achkar JP, McDonald C. ATG16L1 and NOD2 interact in an autophagy-dependent antibacterial pathway implicated in Crohn's disease pathogenesis. Gastroenterology 2010;139:1630-41.

47. Schmid D, Pypaert M, Munz C. Antigen-loading compartments for major histocompatibility complex class II molecules continuously receive input from autophagosomes. Immunity 2007;26:79-92.

48. Cooney R, Baker J, Brain O, et al. NOD2 stimulation induces autophagy in dendritic cells influencing bacterial handling and antigen presentation. Nat Med 2010;16:90-7.

49. Singh SB, Davis AS, Taylor GA, Deretic V. Human IRGM induces autophagy to eliminate intracellular mycobacteria. Science 2006;313:1438-41.

50. McCarroll SA, Huett A, Kuballa P, et al. Deletion polymorphism upstream of IRGM associated with altered IRGM expression and Crohn's disease. Nat Genet 2008;40:1107-12.

51. Brest $\mathrm{P}$, Lapaquette $\mathrm{P}$, Souidi $\mathrm{M}$, et al. A synonymous variant in IRGM alters a binding site for miR-196 and causes deregulation of IRGM-dependent xenophagy in Crohn's disease.

Nat Genet 2011;43:242-5

52. Hashimoto D, Ohmuraya M, Hirota M, et al. Involvement of autophagy in trypsinogen activation within the pancreatic acinar cells. J Cell Biol 2008;181:1065-72.

53. Mareninova OA, Hermann K, French SW, et al. Impaired autophagic flux mediates acinar cell vacuole formation and trypsinogen activation in rodent models of acute pancreatitis. J Clin Invest 2009;119:3340-55.

54. Grasso D, Ropolo A, Lo RA, at al. Zymophagy, a novel selective autophagy pathway mediated by VMP1-USP9x-p62, prevents pancreatic cell death. J Biol Chem 2011;286:8308-24.

55. White E, DiPaola RS. The double-edged sword of autophagy modulation in cancer. Clin Cancer Res 2009;15:5308-16.

56. Yue Z, Jin S, Yang C, Levine AJ, Heintz N. Beclin 1, an autophagy gene essential for early embryonic development, is a haploinsufficient tumor suppressor. Proc Natl Acad Sci USA 2003;100:15077-82.

57. Ding ZB, Shi YH, Zhou J, et al. Association of autophagy defect with a malignant phenotype and poor prognosis of hepatocellular carcinoma. Cancer Res 2008;68:9167-75.

58. Ionov Y, Nowak N, Perucho M, Markowitz S, Cowell JK. Manipulation of nonsense mediated decay identifies gene mutations in colon cancer cells with microsatellite instability. Oncogene 2004;23:639-45.

59. Kim MS, Jeong EG, Ahn CH, Kim SS, Lee SH, Yoo NJ. Frameshift mutation of UVRAG, an autophagy-related gene, in gastric carcinomas with microsatellite instability. Hum Pathol 2008;39:1059-63.

60. Liang C, Feng P, Ku B, et al. Autophagic and tumour suppressor activity of a novel Beclin1-binding protein UVRAG. Nat Cell Biol 2006;8:688-99.

61. Villanueva A, Chiang DY, Newell P, et al. Pivotal role of mTOR signaling in hepatocellular carcinoma. Gastroenterology 2008;135:1972-83.

62. Lang SA, Gaumann A, Koehl GE, et al. Mammalian target of rapamycin is activated in human gastric cancer and serves as a target for therapy in an experimental model. Int J Cancer 2007;120:1803-10.

63. Toso C, Merani S, Bigam DL, Shapiro AM, Kneteman NM. Sirolimus-based immunosuppression is associated with increased survival after liver transplantation for hepatocellular carcinoma. Hepatology 2010;51:1237-43.

64. Degenhardt K, Mathew R, Beaudoin B, et al. Autophagy promotes tumor cell survival and restricts necrosis, inflammation, and tumorigenesis. Cancer Cell 2006;10:51-64.

65. Apel A, Herr I, Schwarz H, Rodemann HP, Mayer A. Blocked autophagy sensitizes resistant carcinoma cells to radiation therapy. Cancer Res 2008;68:1485-94.

66. Cao C, Subhawong T, Albert JM, et al. Inhibition of mammalian target of rapamycin or apoptotic pathway induces autophagy and radiosensitizes PTEN null prostate cancer cells. Cancer Res 2006;66:10040-7.

67. Crighton D, Wilkinson S, O'Prey J, et al. DRAM, a p53-induced modulator of autophagy, is critical for apoptosis. Cell 2006;126:121-34.

68. Amaravadi RK, Yu D, Lum JJ, et al. Autophagy inhibition enhances therapy-induced apoptosis in a Myc-induced model of lymphoma. J Clin Invest 2007;117:326-36. 
69. Chen N, Debnath J. Autophagy and tumorigenesis. FEBS Lett 2010;584:1427-35.

70. Hidvegi T, Schmidt BZ, Hale P, Perlmutter DH. Accumulation of mutant alphal-antitrypsin $\mathrm{Z}$ in the endoplasmic reticulum activates caspases-4 and -12, NFkappaB, and BAP31 but not the unfolded protein response. J Biol Chem 2005;280:39002-15.

71. Teckman JH, Perlmutter DH. Retention of mutant alpha(1)-antitrypsin $\mathrm{Z}$ in endoplasmic reticulum is associated with an autophagic response. Am J Physiol Gastrointest Liver Physiol 2000;279:G961-74.

72. Hidvegi T, Ewing M, Hale P, et al. An autophagy-enhancing drug promotes degradation of mutant alpha1-antitrypsin $\mathrm{Z}$ and reduces hepatic fibrosis. Science 2010;329:229-32.

73. Luciani A, Villella VR, Esposito S, et al. Defective CFTR induces aggresome formation and lung inflammation in cystic fibrosis through ROS-mediated autophagy inhibition. Nat Cell Biol 2010;12:863-75.

74. Maiuri L, Luciani A, Giardino I, et al. Tissue transglutaminase activation modulates inflammation in cystic fibrosis via PPARgamma down-regulation. J Immunol 2008;180:7697-705.

75. Luciani A, Villella VR, Vasaturo A, et al. SUMOylation of tissue transglutaminase as link between oxidative stress and inflammation. J Immunol 2009;183:2775-84.

76. Cardinal J, Pan P, Dhupar R, et al. Cisplatin prevents high mobility group box 1 release and is protective in a murine model of hepatic ischemia/reperfusion injury. Hepatology 2009;50:565-74.

77. Gotoh K, Lu Z, Morita M, et al. Participation of autophagy in the initiation of graft dysfunction after rat liver transplantation. Autophagy 2009;5:351-60.

78. Murry CE, Jennings RB, Reimer KA. Preconditioning with ischemia: A delay of lethal cell injury in ischemic myocardium. Circulation 1986;74:1124-36.
79. Domart MC, Esposti DD, Sebagh M, et al. Concurrent induction of necrosis, apoptosis, and autophagy in ischemic preconditioned human livers formerly treated by chemotherapy. J Hepatol 2009;51:881-9.

80. Azoulay D, Del Gaudio M, Andreani P, et al. Effects of 10 minutes of ischemic preconditioning of the cadaveric liver on the graft's preservation and function: The ying and the yang. Ann Surg 2005;242:133-9.

81. Clavien PA, Selzner M, Rudiger HA, et al. A prospective randomized study in 100 consecutive patients undergoing major liver resection with versus without ischemic preconditioning. Ann Surg 2003;238:843-50.

82. Yan S, Jin LM, Liu YX, Zhou L, Xie HY, Zheng SS. Outcomes and mechanisms of ischemic preconditioning in liver transplantation. Hepatobiliary Pancreat Dis Int 2010;9:346-54.

83. Esposti DD, Domart MC, Sebagh M, et al. Autophagy is induced by ischemic preconditioning in human livers formerly treated by chemotherapy to limit necrosis. Autophagy 2010;6:172-4.

84. Zhang L, Yu J, Pan H, et al. Small molecule regulators of autophagy identified by an image-based high-throughput screen. Proc Natl Acad Sci USA 2007;104:19023-8.

85. Laane E, Tamm KP, Buentke E, et al. Cell death induced by dexamethasone in lymphoid leukemia is mediated through initiation of autophagy. Cell Death Differ 2009;16:1018-29.

86. Krams SM, Martinez OM. Epstein-Barr virus, rapamycin, and host immune responses. Curr Opin Organ Transplant 2008;13:563-8.

87. Sarkar S, Krishna G, Imarisio S, Saiki S, O'Kane CJ, Rubinsztein DC. A rational mechanism for combination treatment of Huntington's disease using lithium and rapamycin. Hum Mol Genet 2008;17:170-8. 


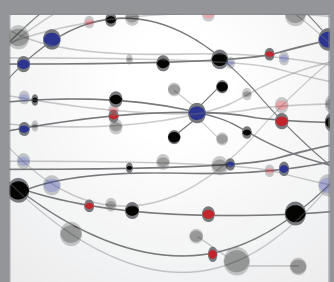

The Scientific World Journal
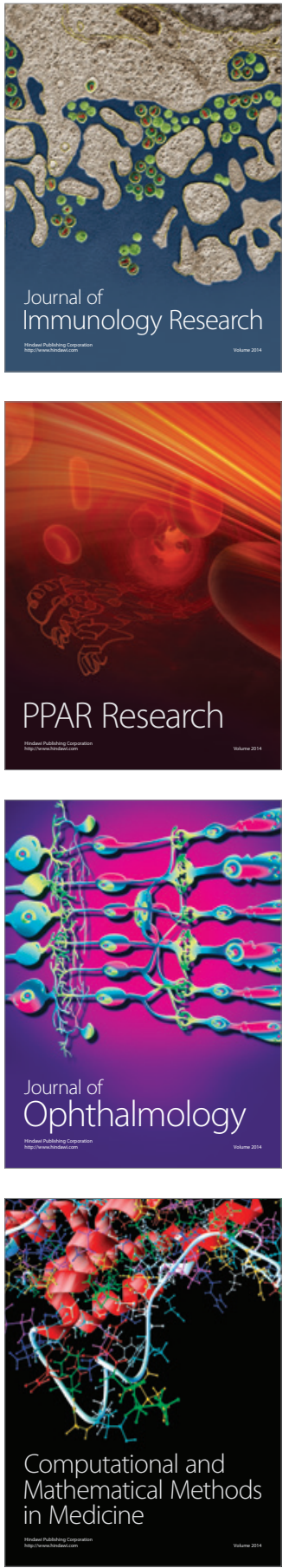

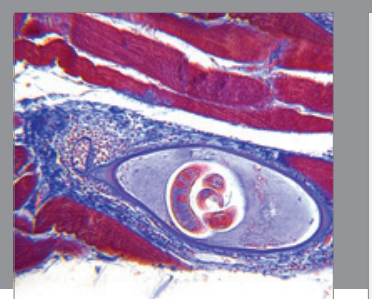

Gastroenterology Research and Practice

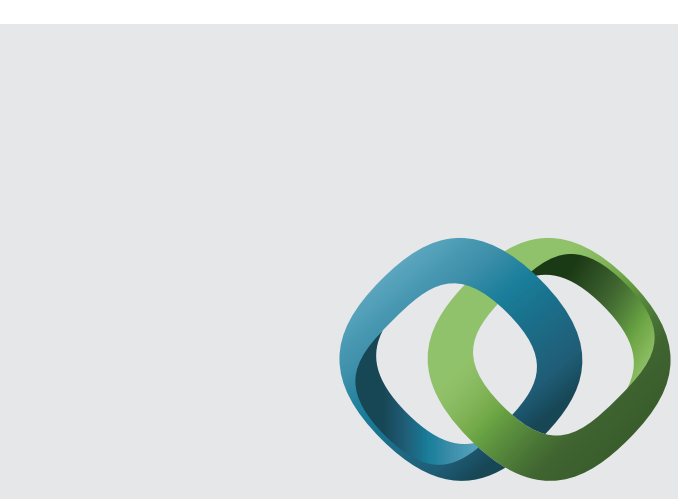

\section{Hindawi}

Submit your manuscripts at

http://www.hindawi.com
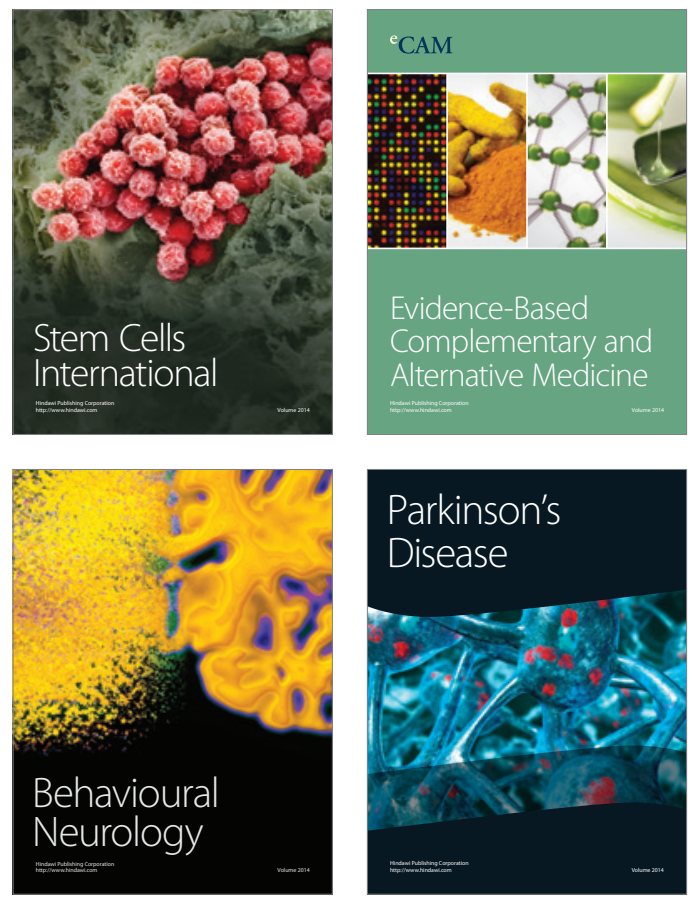
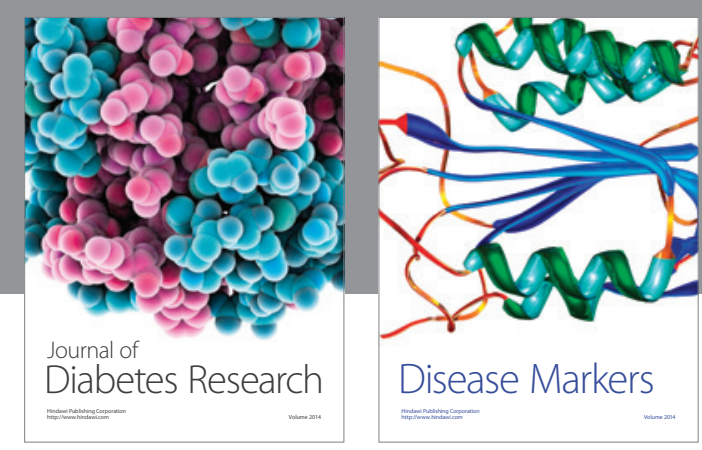

Disease Markers
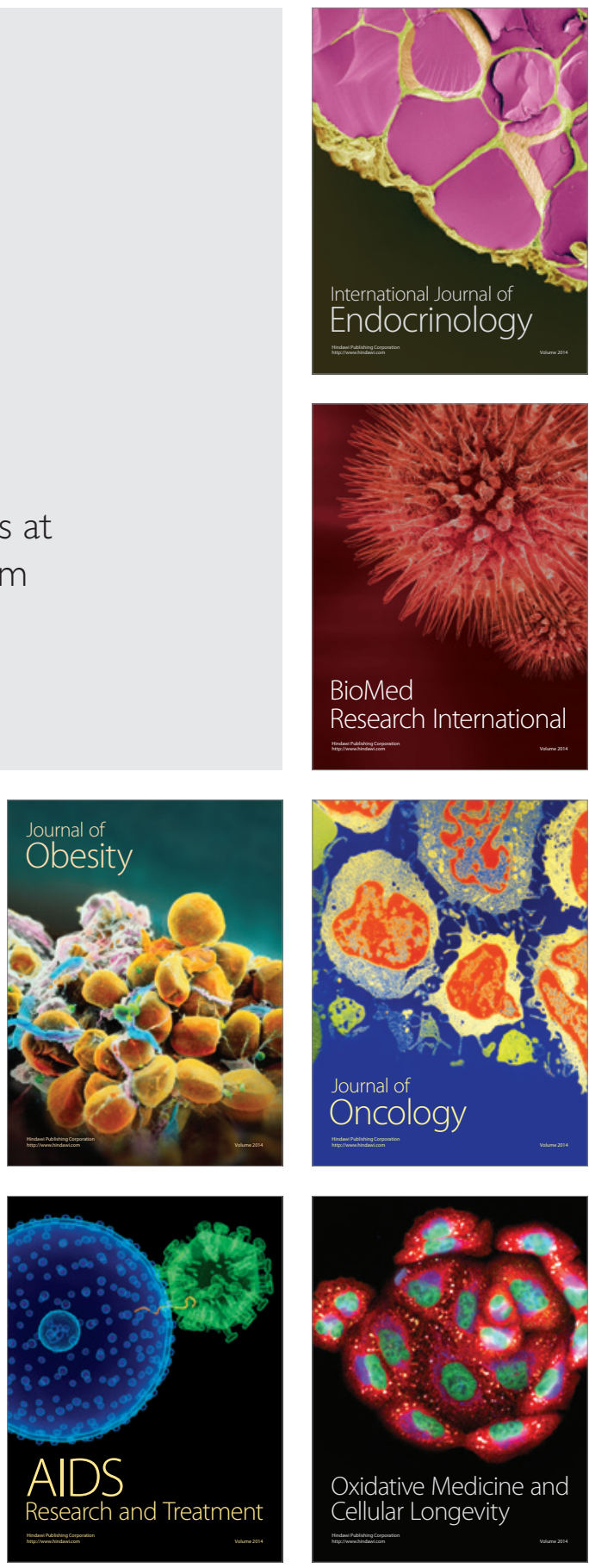